\title{
Tree nut, peanut, and peanut butter consumption and the risk of gastric and esophageal cancer subtypes: the Netherlands Cohort Study
}

\author{
Lisette Nieuwenhuis ${ }^{1}$ (D) Piet A. van den Brandt ${ }^{1,2}$
}

Received: 13 December 2017 / Accepted: 15 March 2018 / Published online: 28 March 2018

(c) The Author(s) 2018

\begin{abstract}
Background Nut consumption has been associated with reduced cancer-related mortality. However, it is unclear whether nut consumption also reduces the risk of esophageal and gastric cancer subtypes. We prospectively investigated the relationship of tree nut, peanut, and peanut butter intake with risk of esophageal squamous cell carcinoma (ESCC), esophageal adenocarcinoma (EAC), gastric cardia adenocarcinoma (GCA), and gastric non-cardia adenocarcinoma (GNCA) in the Netherlands Cohort Study.

Methods In 1986, 120,852 males and females, aged 55-69 years, completed a baseline questionnaire on diet and cancer risk factors. After 20.3 years of follow-up, 133 ESCC, 200 EAC, 191 GCA, and 586 GNCA cases, and 3,720 subcohort members were available for multivariable Cox regression analyses, using a case-cohort approach.

Results Increased total nut consumption was significantly associated with a decreased risk of ESCC and GNCA [HRs (95\% CIs) for $10+\mathrm{g}$ /day vs. nonconsumers $=0.54(0.30-0.96)$ and $0.73(0.55-0.97)$, respectively], but not with EAC and GCA risk. Similar trends were observed for tree nut and peanut intake, which were mostly nonsignificant. For peanut butter intake, no significant associations were found. When excluding the first four years of follow-up to reduce the possible influence of reversed causation, the relation between nut consumption and ESCC risk attenuated, but remained inverse.

Conclusions Our findings suggest that increased tree nut and peanut consumption is inversely associated with GNCA risk and possibly with ESCC risk, but not with the risk of the other esophageal and gastric cancer subtypes.
\end{abstract}

Keywords Chemoprevention $\cdot$ Cohort studies $\cdot$ Esophageal neoplasms $\cdot$ Nuts $\cdot$ Stomach neoplasms

\section{Introduction}

In the past few years, the interest in nuts has been increasing because of their perceived health benefits. Besides other health advantages, recent meta-analyses have demonstrated

Electronic supplementary material The online version of this article (https://doi.org/10.1007/s10120-018-0821-2) contains supplementary material, which is available to authorized users.

Lisette Nieuwenhuis

1.nieuwenhuis@maastrichtuniversity.nl

1 Department of Epidemiology, Care and Public Health Research Institute (CAPHRI), Maastricht University Medical Centre, Maastricht, The Netherlands

2 Department of Epidemiology, GROW - School for Oncology and Developmental Biology, Maastricht University Medical Centre, Maastricht, The Netherlands that increased nut consumption may lower the risk of cancer and cancer-related mortality [1-4]. Nuts are nutrient-dense foods and contain vitamins, minerals, mono- and polyunsaturated fatty acids, polyphenols, and several other compounds that might act as cancer-chemopreventive agents $[5,6]$.

Currently, little evidence is available on the relation between nut consumption and the risk of esophageal and gastric cancer, while these two cancers were the sixth (esophageal) and third (gastric) most common causes of death from cancer globally in 2012 [7]. Based on histologic and topographic subtyping, esophageal and gastric cancer can be subdivided into esophageal squamous cell carcinoma (ESCC), esophageal adenocarcinoma (EAC), gastric cardia adenocarcinoma (GCA), and gastric non-cardia adenocarcinoma (GNCA). A growing number of studies indicates that these subtypes differ regarding their etiologies and risk factors $[8,9]$. Special interest is in EAC and GCA, because the incidence rates of these subtypes have increased 
considerably in the US and in many European countries in the past decades $[10,11]$.

In a recently published prospective cohort study, nut and peanut butter consumption was significantly inversely associated with GNCA risk, but not with risk of the other esophageal and gastric cancer subtypes [12]. Unfortunately, the authors could not analyze types of nuts separately. Moreover, results from four case-control studies investigating the association between nut consumption and gastric cancer are inconclusive [13-16], and one case-control study found an inverse association between peanut consumption and ESCC risk [17]. Another case-control study observed no association between fruit and nut consumption combined and upper aerodigestive tract cancer risk [18].

In this study, we prospectively investigated the associations of tree nut, peanut, and peanut butter consumption with the risk of esophageal and gastric cancer subtypes in the Netherlands Cohort Study (NLCS). Moreover, we investigated the exposure-response curves and whether the associations were modified by sex, alcohol consumption, cigarette smoking, educational level, and body mass index (BMI).

\section{Methods}

\section{Study design and cancer follow-up}

In this analysis, data from the NLCS were used. In September 1986, 62,573 females and 58,279 males, aged 55-69 years, completed a mailed, self-administered baseline questionnaire on cancer risk factors [19]. The institutional review boards from the Maastricht University and the Netherlands Organization for Applied Scientific Research approved the NLCS. By filling in and returning the baseline questionnaire, participants agreed to participate in the study.

For data processing and analysis, a case-cohort approach was used for efficiency reasons. A subcohort $(n=5,000)$ was randomly sampled from the total cohort immediately after baseline, and accumulated person-years were estimated from this subcohort. Vital status information of subcohort members was obtained biannually from 17 September 1986 until 1 January 2007, and was 100\% complete after 20.3 years. Incident cancer cases in the total cohort were detected through annual record linkage with the Netherlands Cancer Registry and the nationwide Dutch Pathology Registry (PALGA) [20]. The completeness of cancer incidence follow-up is estimated to be more than $95 \%$ [21].

During the 20.3 years of follow-up, 164 ESCC, 259 EAC, 254 GCA, and 741 GNCA cases without prevalent cancer (except skin cancer) at baseline were detected. Histology codes for esophageal cancer (ICD-O-3 code C15) included 8050-8076 for ESCC, and 8140-8141, 8190-8231, 8260-8263, 8310, 8430, 8480-8481, 8490, 8560, and 8570-8572 for EAC. For gastric cancer (ICD-O-3 code C16), histology codes were C16.0 for GCA and C16.1-16.9 for GNCA. Participants with incomplete or inconsistent dietary data were excluded from the analysis, as were participants with missing values on predefined confounders. In the current analysis, $133 \mathrm{ESCC}, 200 \mathrm{EAC}, 191 \mathrm{GCA}$, and 586 GNCA cases, and 3,720 subcohort members were included (Fig. 1).

\section{Exposure assessment}

The 11-page mailed, self-administered baseline questionnaire consisted of questions regarding diet, smoking habits,
Fig. 1 Flow diagram of the number of subcohort members and gastric and esophageal cancer cases on whom the analyses were based

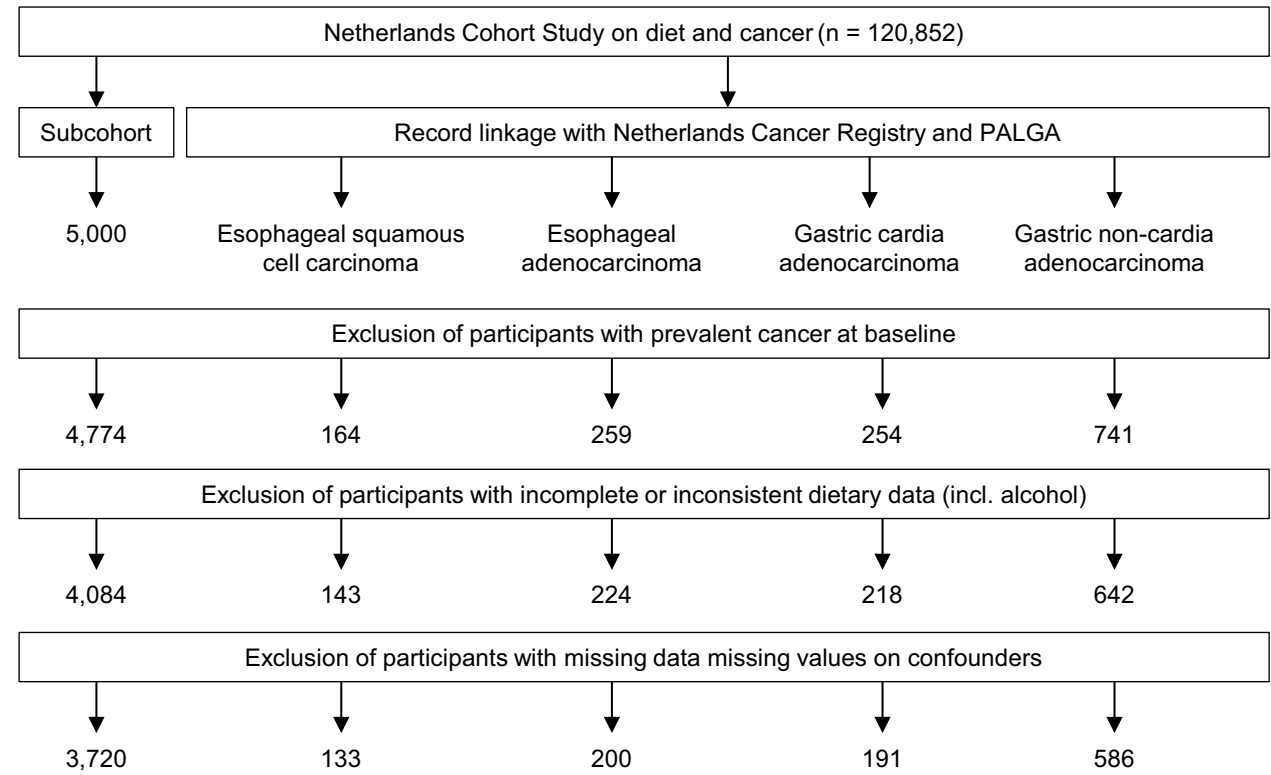


anthropometry, disease history, physical activity, and other cancer risk factors [19]. Information on diet was obtained from a validated 150 -item semi-quantitative food-frequency questionnaire (FFQ) that asked about habitual diet in the year before baseline [22]. Intake of tree nuts, peanuts, and peanut butter was assessed by measuring the number of standard portion sizes consumed per intake and the intake frequencies of 'peanuts', 'other, mixed nuts' (tree nuts), and 'peanut butter'. Frequency categories could range from 'never or less than $1 \times /$ month' to ' $6-7 \times /$ week'. A standard portion size of peanuts or tree nuts was assumed to be $28 \mathrm{~g}$, and one portion of peanut butter $15 \mathrm{~g}$ per slice of bread. Mean daily intake was calculated in grams, by multiplying intake frequencies and portion sizes. Total nut consumption was calculated as the sum of tree nut and peanut intake.

\section{Statistical analysis}

The associations between nut intake and risk of esophageal and gastric cancer subtypes were analyzed with age- and sex-adjusted and multivariable-adjusted Cox proportional hazards models. The robust Huber-White sandwich estimator was used to estimate standard errors that take into account the additional variance introduced by sampling from the total cohort [23]. The proportional hazards assumption was checked with Schoenfeld residuals and $-\ln (-\ln )$ survival plots [24]. If the assumption was violated for a variable, the interaction between that variable and time was tested by including a time-varying covariate in the model.

The associations between nut and peanut butter intake and risk of esophageal and gastric cancer subtypes were investigated on a categorical and continuous scale in survival analyses. We combined both sexes in the categorical analyses, because of the limited number of cases and because no statistically significant interaction by sex was found (Online Resource 2). In the continuous analyses, we additionally estimated hazard rations (HR) for males and females separately. For the categorical analyses, total nut and peanut consumption was divided into four categories of $0,0.1-<5,5-<10$, and $10+\mathrm{g} /$ day. Due to the smaller number of cases in the higher intake categories, tree nut consumption was categorized into 0 and $0.1+\mathrm{g} /$ day, and peanut butter into $0,0.1-<5$, and $5+\mathrm{g} /$ day. The category of nonconsumers formed the reference group. Tests for trends were performed by assigning sex-specific median values of nut intake in the subcohort to the intake categories and fitting these as continuous terms in the regression models. In the continuous analyses, HRs were estimated per increment of $5 \mathrm{~g} /$ day.

In multivariable-adjusted survival analyses, the associations were corrected for the following predefined confounders, which were included in the final multivariable-adjusted model independent of their effect on the estimated HRs: age at baseline (years; continuous), sex (male/female), cigarette smoking [status (never, former, current), frequency (number of cigarettes per day; continuous, centered), and duration (years; continuous, centered)], BMI $(<18.5,18.5-<25$, $25-<30$, and $30+\mathrm{kg} / \mathrm{m}^{2}$ ), nonoccupational physical activity $(\leq 30,>30-\leq 60,>60-\leq 90,>90 \mathrm{~min} /$ day $)$, highest level of education [primary school or lower vocational (low), secondary school or medium vocational (medium), and higher vocational or university (high)], total energy intake (kcal/ day; continuous), alcohol consumption $(0,0.1-<5,5-<15$, $15-<30$, and $30+\mathrm{g} /$ day), and family history of esophageal (for esophageal cancer subtypes) or gastric cancer (for gastric cancer subtypes). Other potential confounders considered were: intake of fruits, vegetables, tea, coffee, red meat, processed meat, fish, and total salt, history of gastric ulcers, and long-term use ( $>6$ months) of nonsteroidal antiinflammatory drugs (including aspirin) and lower esophageal sphincter-relaxing medications. However, because these potential confounders did not change the HRs with $10 \%$ when using a backward stepwise selection procedure, only the predefined confounders were included in the final multivariable-adjusted model.

Tests for heterogeneity were performed to investigate etiologic differences between the four major subtypes of esophageal and gastric cancer using a competing risk procedure. In this analysis, a bootstrapping method developed for the case-cohort approach was used to estimate the standard errors for the observed differences in associations [25, 26].

The linearity of the exposure-response relation between nut intake and risk of esophageal and gastric cancer subtypes was assessed in restricted cubic spline analysis, in models with three fixed knots at 0,5 , and $10 \mathrm{~g}$ nut intake/ day. Because no assumptions are made about the shape of the relation between the exposure and outcome variables in restricted cubic spline analysis, it is a useful method to test for nonlinearity and to present nonlinear relationships. Detailed sensitivity analyses were performed to investigate whether choosing additional knots or other knot positions would improve the model fit, as measured with the Akaike Information Criterion (AIC) score [27].

To investigate possible interactions by esophageal and gastric cancer risk factors, categorical analyses of nut consumption were performed stratified by sex, baseline BMI, smoking status, alcohol consumption, and educational level. To increase statistical power, the two highest nut intake categories were merged. Interactions were tested by including cross-product terms in the models and performing Wald tests.

To check for potential reversed causation due to preclinical cancer at baseline, we divided the total follow-up time into 4-year periods and compared the median nut intake at baseline of cases diagnosed during these periods. A Kruskal-Wallis test was performed to test the statistical 
significance of a possible difference in median nut intake. Moreover, we repeated the Cox regression analyses after excluding the first 4 years of follow-up. Additionally, we restricted the analyses to cases who had stated having had a constant peanut butter intake during the 5 years before baseline. We do not have these data for tree nut or peanut consumption.

In sensitivity analyses, we additionally adjusted for adherence to the Mediterranean diet, by including the alternate Mediterranean diet score (aMed) into the regression models [28]. Since nuts comprise one of the components of the aMed score, and because alcohol consumption is positively associated with esophageal and gastric cancer, an adapted version was used (excluding nuts and alcohol), which ranged from 0 (no adherence) to 7 (maximal adherence).

All analyses were performed in Stata 14 software (StataCorp. 2015. College Station, TX). $P$ values were tested two sided, and were considered statistically significant if $p<0.05$.

\section{Results}

Table 1 shows the baseline characteristics of subcohort members and cases for males and females separately. All cases, except EAC cases, consumed on average less total nuts and tree nuts than subcohort members in both sexes. Male subcohort members consumed less peanuts than esophageal cancer cases, but more than GNCA cases. Female subcohort members consumed more peanuts than all cases, except EAC cases. Moreover, in males, subcohort members consumed more peanut butter than gastric cancer cases, but less than esophageal cancer cases. In females, subcohort members consumed more peanut butter than cases.

Cases and subcohort members also differed with respect to other, potentially confounding factors: compared to subcohort members, cases of both sexes were older (except for male EAC and GCA cases), more often ever smokers (except for female EAC cases), and less educated (except for GCA cases). EAC and GCA cases of both sexes and female GNCA cases were heavier than subcohort members. Moreover, male cases more often reported a positive family history of gastric cancer than subcohort members, and female cases more often reported gastric ulcers. Furthermore, male cases consumed more alcohol, red and processed meat, and coffee than subcohort members. Female cases also consumed more red meat and coffee than subcohort members.

In Table 2, multivariable-adjusted associations between nut and peanut butter consumption and risk of esophageal and gastric cancer subtypes are presented. The proportional hazards assumption was potentially violated for some categories of total nut and peanut consumption in the analyses of ESCC and GNCA risk, and for peanut butter consumption in the analyses of ESCC and EAC risk. Ageand sex-adjusted results are presented in Online Resource 1 , which were not importantly different from the multivariable-adjusted results; positive associations became slightly stronger, whereas inverse associations became somewhat weaker after multivariable adjustment.

For total nut consumption, a statistically significant inverse relation was found with ESCC risk: the HR $(95 \%$ CI) for those consuming $10+\mathrm{g} /$ day versus nonconsumers was $0.54(0.30-0.96)\left(P_{\text {trend }}=0.050\right)$. Increased total nut intake was non-significantly positively related to EAC risk $\left(P_{\text {trend }}=0.578\right)$, and, in continuous analyses, a significant positive association with EAC risk was found in women only [HR $(95 \% \mathrm{CI})$ per $5 \mathrm{~g} /$ day increment $=1.19$ (1.07-1.32)]. No clear relation with total nut intake was seen for GCA risk. For GNCA risk, a nonsignificant inverse trend was found $\left(P_{\text {trend }}=0.088\right)$, with significant inverse associations in all total nut consumption categories: the HRs $(95 \% \mathrm{CI})$ for those consuming $0.1-<5$, $5-<10$, and $10+\mathrm{g}$ total nuts/day vs. nonconsumers were 0.79 (0.63-0.98), 0.62 (0.44-0.87), and 0.73 (0.55-0.97), respectively. No significant interaction between total nut consumption categories and sex was observed for the esophageal and gastric cancer subtypes $\left(P_{\text {interaction }} \geq 0.206\right)$ (Online Resource 2). In sensitivity analyses, additional adjustment for the aMed score did not importantly alter the results (data not shown).

The heterogeneity test for the relation of categorical total nut intake with risk of esophageal and gastric cancer subtypes was significant $\left(P_{\text {heterogeneity }}=0.008\right)$. To further investigate this finding, we compared the subtypes pairwise. Significant differences in associations were only found between EAC and GNCA $\left(P_{\text {heterogeneity }}=0.004\right)$ and between GCA and GNCA ( $\left.P_{\text {heterogeneity }}=0.049\right)$. A borderline significant difference was observed between ESCC and EAC $\left(P_{\text {heterogeneity }}=0.050\right)$.

Tree nut intake was significantly associated with a lower risk of ESCC (HR (95\% CI) for consumers vs. nonconsumers $=0.56(0.35-0.90)$. For EAC risk, a nonsignificant positive association was found. Tree nut consumption was nonsignificantly inversely related to GCA risk, and the HR $(95 \%$ CI) per $5 \mathrm{~g}$ /day increment was $0.23(0.06-0.90)$ in women in continuous analyses. For GNCA risk, also a nonsignificant inverse association was observed, and, in continuous analyses, significant inverse associations in the overall population and in women: the HRs (95\% CI) per $5 \mathrm{~g}$ /day increment were $0.81(0.66-0.99)$ and $0.52(0.33-0.81)$, respectively.

For peanut intake, nonsignificant inverse associations with risk of ESCC and GNCA were found, and a HR (95\% $\mathrm{CI})$ for $\mathrm{GNCA}$ for those consuming $5-<10 \mathrm{~g}$ peanuts/day compared to nonconsumers of $0.69(0.48-0.99)$. The risk of EAC was non-significantly increased in participants with a higher peanut intake, and this association was significant in 


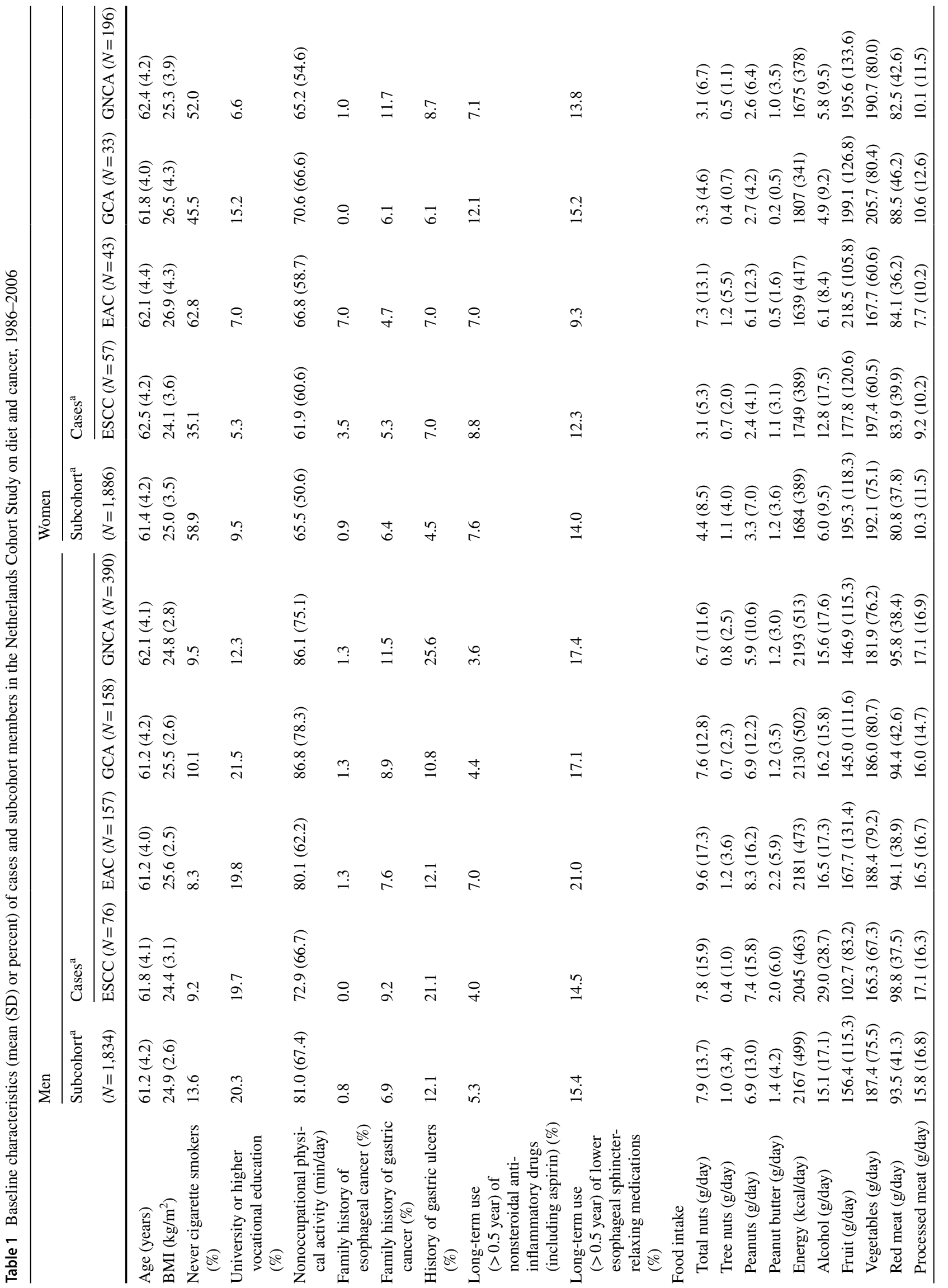


women in continuous analyses [HR $(95 \% \mathrm{CI})$ per $5 \mathrm{~g}$ /day increment $=1.20(1.07-1.35)]$. For GCA risk, no association with peanut intake was found.

Increasing peanut butter intake was associated with a nonsignificantly increased risk of ESCC and EAC. In continuous analyses, the association with ESCC risk was significant in the overall population and in men [HR $(95 \% \mathrm{CI})=1.25$ (1.03-1.25) and 1.33 (1.06-1.67), respectively], as was the association with EAC risk in men [HR (95\% CI) per $5 \mathrm{~g} /$ day increment $=1.19(1.03-1.37)]$. Unclear associations were found between peanut butter consumption and GCA and GNCA risk, although a significant inverse association with GCA risk was observed in women in continuous analyses $[\mathrm{HR}(95 \% \mathrm{CI})$ per $5 \mathrm{~g} /$ day increment $=0.08(0.01-0.77)]$.

Figure 2 presents the restricted cubic spline curves with three fixed knots at 0,5 , and $10 \mathrm{~g}$ nut intake/day for the risk of (a) ESCC, (b) EAC, (c) GCA, and (d) GNCA according to total nut consumption. A clear leveling-off of ESCC, GCA, and GNCA risk can be seen for total nut intake of more than $5 \mathrm{~g} /$ day. Statistical evidence for nonlinearity was only found for GNCA $\left(P_{\text {nonlinearity }}=0.001\right)$. For tree nut, peanut, and peanut butter intake, the test for nonlinearity was solely significant for the relation between peanut consumption and GNCA risk $\left(P_{\text {nonlinearity }}=0.013\right)$. Choosing additional knots or other knot positions did not improve the fit of the model, as measured with the AIC score (data not shown).

In the stratified analyses, we observed no significant interactions between total nut consumption categories and potential risk factors for ESCC, GCA, and GNCA (Online Resource 2). For EAC, we found a significant interaction between total nut intake and educational level $\left(P_{\text {interaction }}=0.043\right)$, with positive associations in the subgroups with a low or medium educational level, and an unclear trend in the subgroup with a high educational level (Online Resource 3). However, because of the low case numbers and the large number of Wald tests performed, this finding may be due to chance.

The median total nut intake at baseline of ESCC cases diagnosed earlier in the follow-up was lower than that of cases diagnosed later in time (Online Resource 4), and the Kruskal-Wallis test showed that there was a significant difference between the 4 -year periods $(P=0.019)$. For GNCA, the median intake was higher in earlier versus later cases, although not significantly $(P=0.077)$. For EAC and GCA cases, no significant differences were observed.

Table 3 presents the multivariable-adjusted HRs for risk of esophageal or gastric cancer subtypes according to nut intake after excluding the first 4 years of follow-up. When excluding the first 4 years of follow-up, most inverse associations of categorical nut and peanut butter intake with ESCC risk attenuated, e.g., the HR $(95 \% \mathrm{CI})$ for ESCC for $0.1-<5 \mathrm{~g}$ total nut intake/day vs. nonconsumers changed from $0.81(0.52-1.24)$ to $1.05(0.66-1.66)$, and that for 


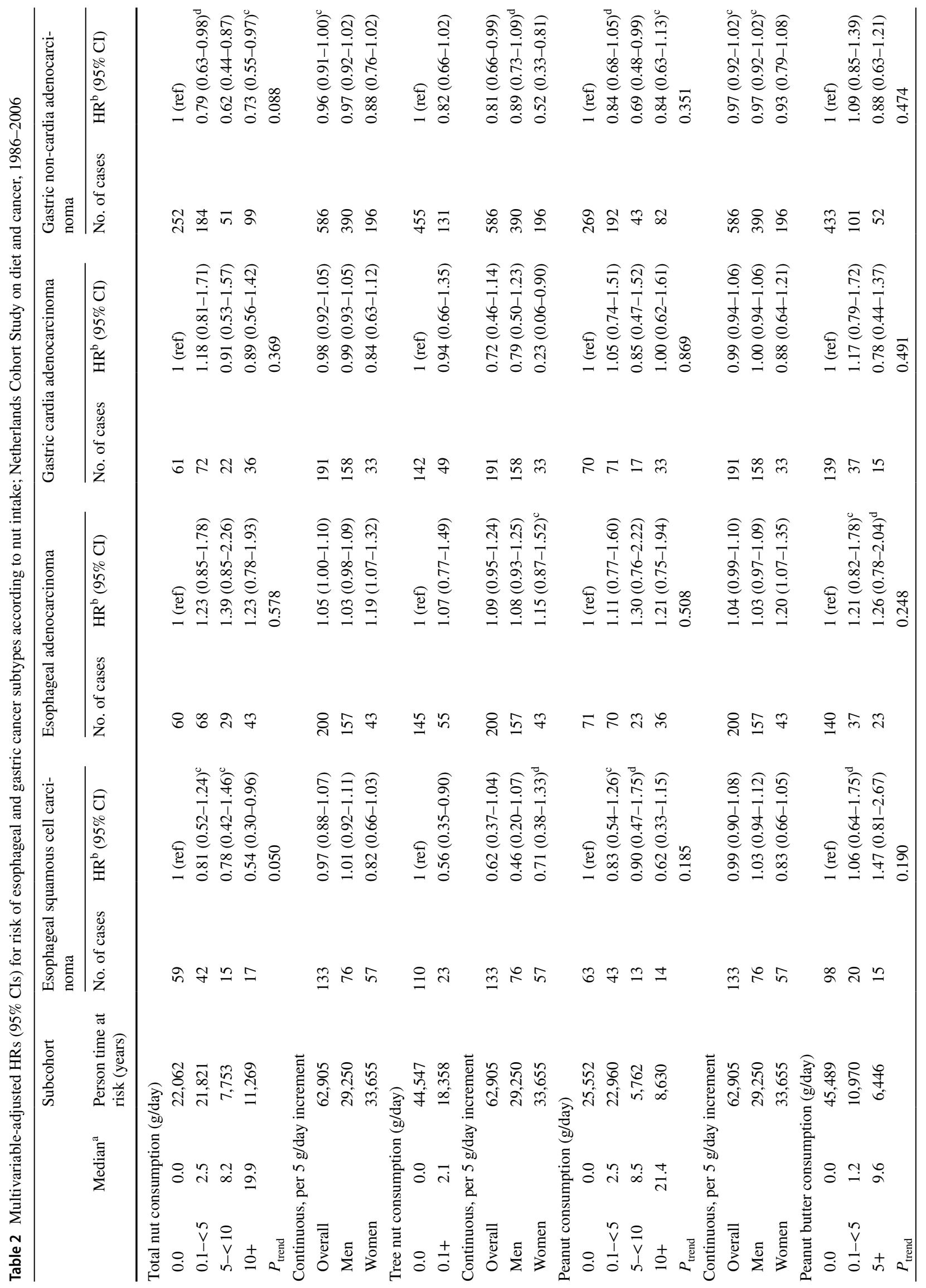




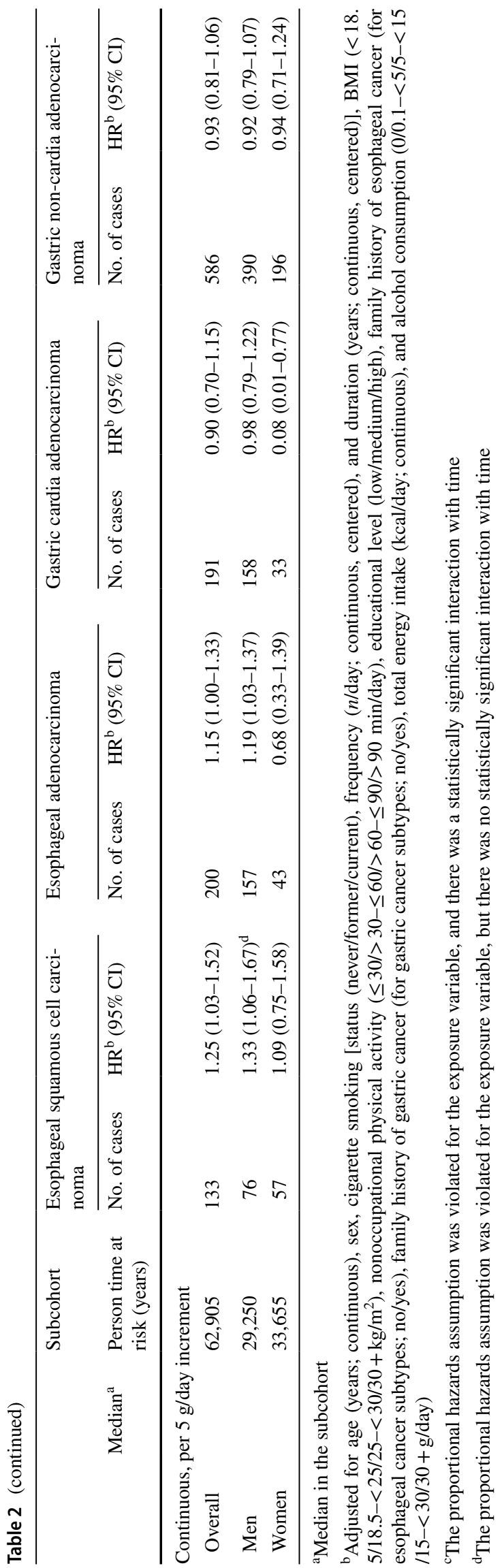

$5-<10 \mathrm{~g}$ total nut consumption/day vs. nonconsumers from $0.78(0.42-1.46)$ to $0.95(0.49-1.84)$. For EAC, GCA, and GNCA risk, the associations were essentially the same as when the total follow-up period was included. Restricting the analysis of peanut butter to only those participants with a constant peanut butter intake during the 5 years before baseline did not importantly change the results (data not shown).

\section{Discussion}

In this prospective cohort study, increased total nut consumption was significantly associated with a decreased risk of ESCC and GNCA. A nonsignificant positive association was observed with EAC risk and no clear relation with GCA risk. Similar trends were found for tree nut and peanut intake, which were mostly nonsignificant. For peanut butter intake, nonsignificant positive associations were found with the esophageal cancer subtypes and unclear associations with the gastric cancer subtypes. Statistical evidence for nonlinearity was only observed for the relation between total nut consumption and GNCA risk. Moreover, no significant interactions between total nut consumption and risk factors for esophageal and gastric cancer subtypes were identified. When excluding the first 4 years of follow-up to reduce the influence of potential reversed causation, conclusions remained the same for EAC, GCA, and GNCA risk. The associations between nut consumption and ESCC risk attenuated, but remained inverse.

Our results are partially in accordance with a recent publication from the prospective NIH-AARP Diet and Health Study in the US [12]. In this US cohort, nut consumption was also associated with a significantly reduced GNCA risk during a median follow-up period of 15.5 years, but no relation was found with ESCC risk. Unfortunately, they did not investigate different nut types separately. Moreover, they observed a significant inverse association between peanut butter consumption and GNCA risk, while we found no significant relations with peanut butter. This difference may be due to the higher mean (SD) peanut butter intake in the US cohort [3.0 (7.4) g/day] than in our study [1.3 (3.9) g/ day]. Another possible explanation is the higher number of cases in the US cohort and thus the higher statistical power. In addition, we observed that higher consumption of nuts and peanut butter was non-significantly associated with an increased EAC risk. The positive relation for nut intake is an unexpected finding, for which we do not have a clear explanation. This result is not consistent with the results from the NIH-AARP Diet and Health Study, because they found no association between nut intake and EAC risk [12]. No other studies investigated this association and, therefore, further 

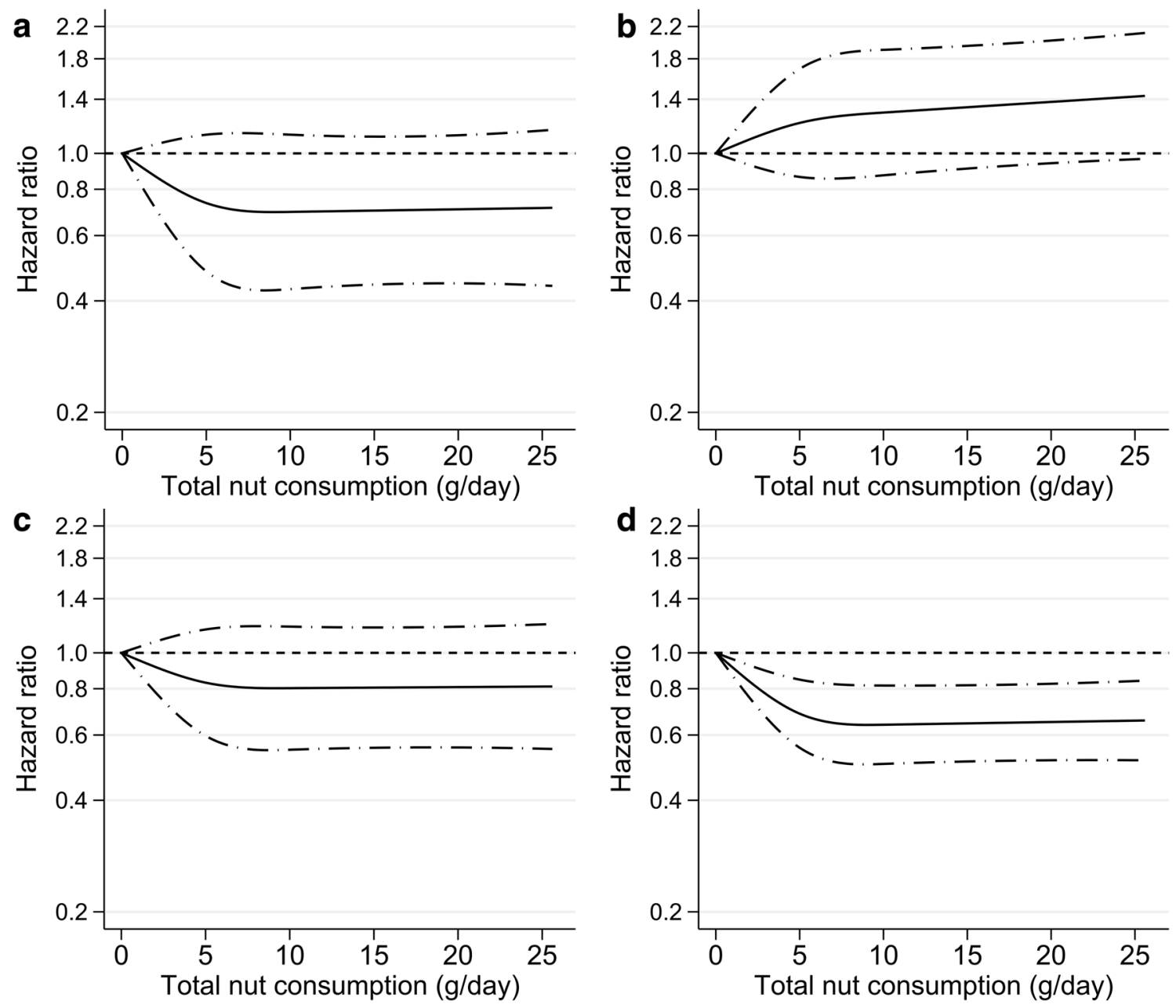

Fig. 2 Multivariable-adjusted restricted cubic spline curves for the association between total nut consumption ( $\mathrm{g} /$ day) and risk of a esophageal squamous cell carcinoma (ESCC), b esophageal adenocarcinoma (EAC), c gastric cardia adenocarcinoma (GCA), and $\mathbf{d}$ gastric non-cardia adenocarcinoma (GNCA). Solid lines represent point estimates; dashed lines represent $95 \%$ CIs. $P$ values for nonlinearity were 0.193 for ESCC, 0.394 for EAC, 0.299 for GCA, and 0.001 for GNCA. HRs were adjusted for age (years; continuous), sex, ciga-

research regarding the relation between nut consumption and EAC risk is required.

Results from several case-control studies are inconclusive: for gastric adenocarcinoma risk, one case-control study found a significant positive trend with frequency of nut consumption [13], two observed significant inverse associations when comparing cases to population controls $[15,16]$, and one found no association with total nut consumption [14]. For ESCC, a significant inverse association was observed with peanut consumption frequency in one case-control study [17]. In another case-control study, no relation was seen between fruit and nut consumption combined and risk of aerodigestive tract cancer [18]. These

rette smoking [status (never/former/current), frequency (n/day; continuous, centered), duration (years; continuous, centered)], BMI $(<1$ $\left.8.5 / 18.5-<25 / 25-<30 / 30+\mathrm{kg} / \mathrm{m}^{2}\right)$, nonoccupational physical activity $(\leq 30 />30-\leq 60 />60-\leq 90 />90 \mathrm{~min} /$ day), educational level (low/ medium/high), family history of esophageal cancer (for esophageal cancer subtypes; no/yes), family history of gastric cancer (for gastric cancer subtypes; no/yes), total energy intake (kcal/day; continuous), and alcohol consumption $(0 / 0.1-<5 / 5-<15 / 15-<30 / 30+$ g/day $)$

contradicting results may be caused by the small sample sizes of the studies, and due the fact that these studies were vulnerable to selection and information biases, a problem intrinsic to all case-control studies.

Reversed causation may be another explanation for the inconsistent findings in the above-described case-control studies. People with esophageal or gastric cancer may already suffer from symptoms related to their disease before diagnosis. These prediagnostic complaints may result in changes in diet and, consequently, in biased recall of previous normal dietary habits; this phenomenon has been described previously [29]. Asking participants about their diet long before the onset of symptoms would not solve this 


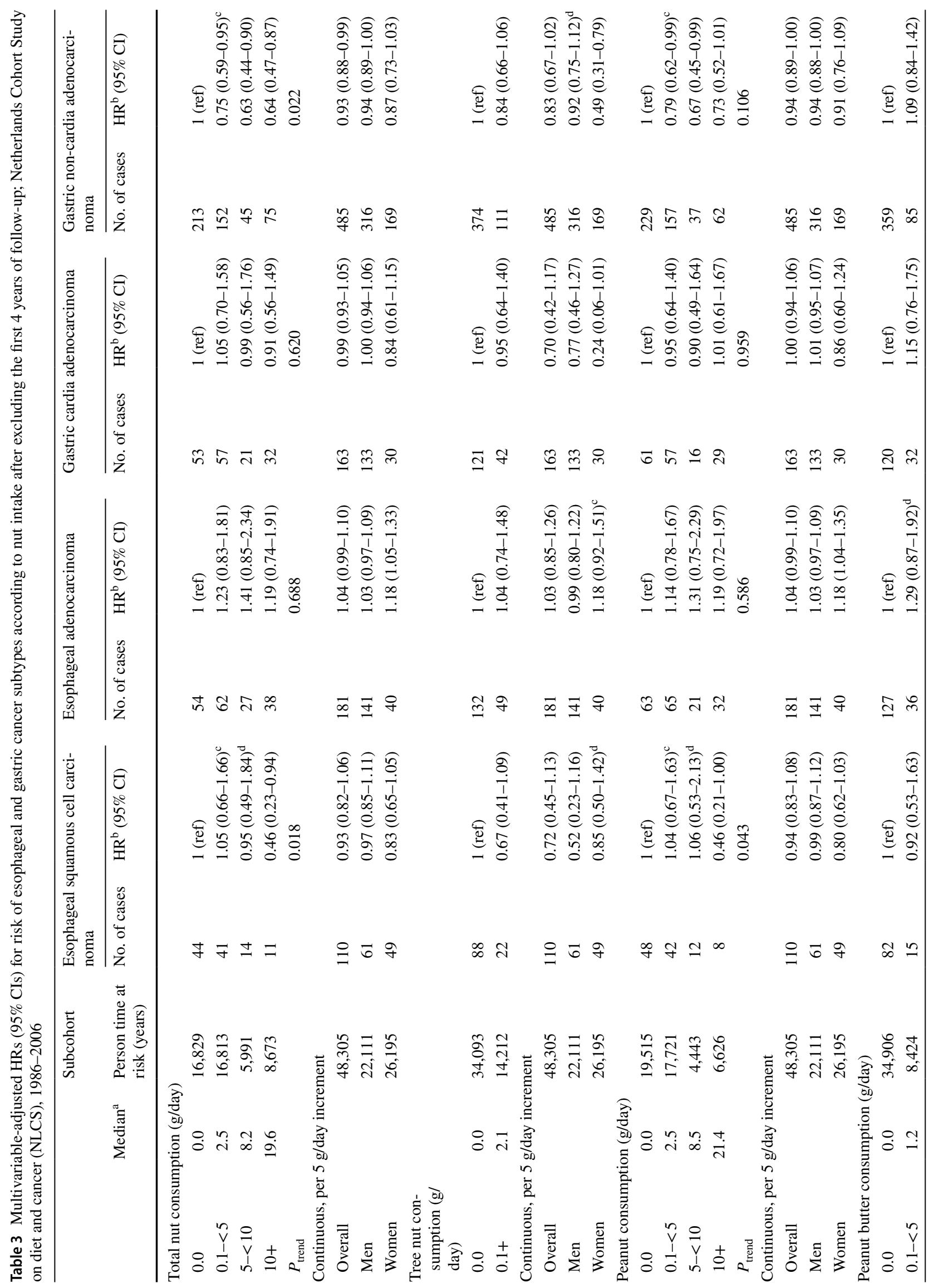




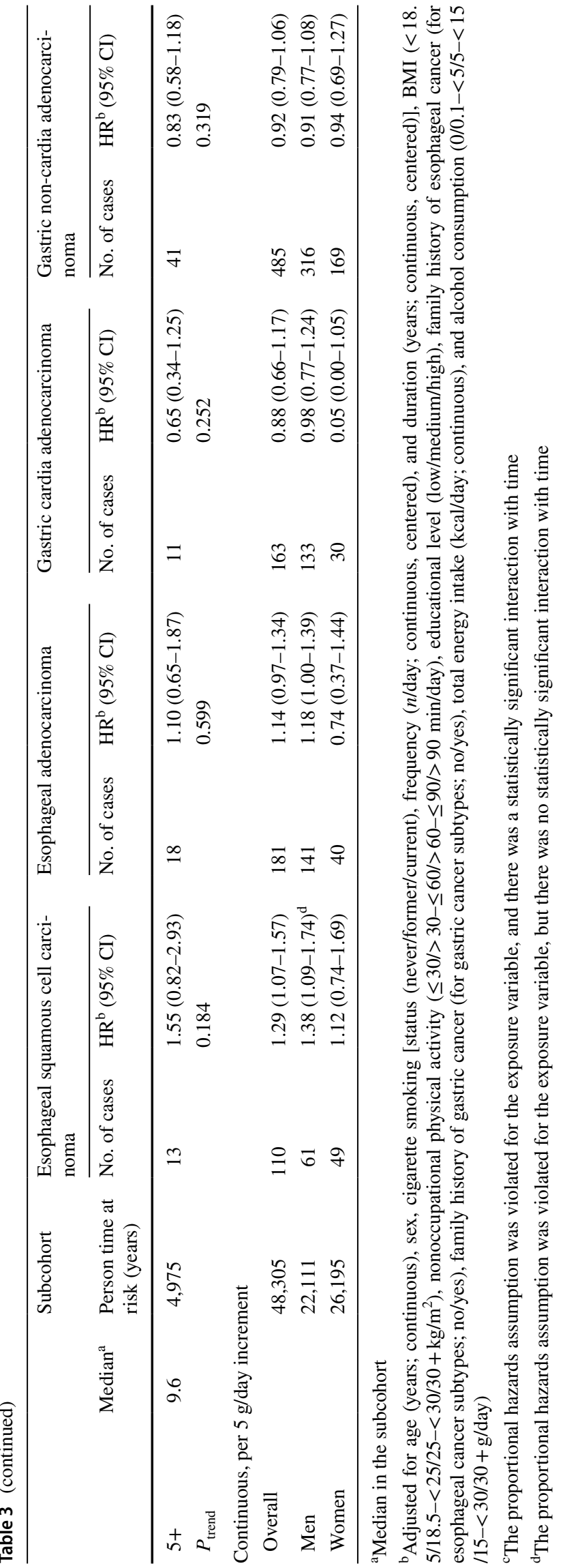

problem, since recall of remote diet is strongly influenced by current diet [30]. In general, cohort studies are less vulnerable to reversed causation than case-control studies because of the longitudinal design. In line with this reasoning, we observed in our study that the median nut consumption significantly differed between ESCC cases diagnosed after different follow-up durations. To investigate the effect of potential reversed causation on our results, we conducted sensitivity analyses in which we excluded the first 4 years of follow-up. This did not change the conclusions about the relation of nut and peanut butter consumption with EAC, GCA, and GNCA risk. The associations between nut consumption and ESCC risk attenuated, but remained inverse. This clearly underlines the importance of taking into account possible information bias due to reversed causation, especially in case-control studies.

Nuts are rich sources of vitamins (e.g., B6, B9, and E), minerals (e.g., selenium and magnesium), fiber, proteins, mono- and polyunsaturated fatty acids, phytosterols, and polyphenols [e.g., flavonoids (quercetin, genistein), stilbenes (resveratrol), and ellagic acid] [5, 6]. However, the nutrient composition varies between nut types. Peanuts, which are botanically legumes, contain comparable amounts of total fat, protein, and fiber as almonds, but higher amounts of saturated fatty acids, folate and phytosterols [31]. Walnuts contain less protein, fiber, and folate than peanuts, and more total fat, which is mainly due to its high polyunsaturated fatty acid content [31]. Peanut butter contains the beneficial components of peanuts, although some additives are supplemented to enhance its quality, taste, and presentation [32]. The main proposed mechanisms by which nuts might conduct their cancer-chemopreventive effects relate to their antioxidant and anti-inflammatory effects [5, 6]. Other hypothesized mechanisms are the regulation of cell differentiation, proliferation and apoptosis, inhibition of tumor initiation, modulation of angiogenesis, induction of DNA damage repair and detoxifying metabolic enzymes, modification of hormonal mechanisms, and alteration of lipid profiles and cell metabolism [5, 6].

EAC mainly develops in the distal esophagus and GCA in the gastric cardia. Therefore, it might be difficult to determine whether a large tumor near the gastroesophageal junction has an esophageal or a gastric origin [33]. Consequently, misclassification of EAC and GCA tumors might occur. In the NLCS, information on cancer incidence was obtained from the Netherlands Cancer Registry. This registry combines pathology and clinical information to obtain high-quality data regarding the topography and histology of tumors, which has been reported to be of high accuracy [34].

The number of esophageal cancer and GCA cases in our study was fairly limited, because of the relatively low incidence of these cancers in the Netherlands [35]. Nevertheless, the large size of the NLCS and the long follow-up of 
20.3 years enabled us to analyze the esophageal and gastric cancer subtypes separately. Due to limited power, we could not perform the analysis for males and females separately, except for the continuous analyses. In the continuous analyses, the associations appeared to be stronger in females than in males. Therefore, we recommend to investigate sexspecific relations in future studies. Another strength is that we were able to investigate the effects of different types of nuts on the risk of esophageal and gastric cancer subtypes. Moreover, the prospective design and the high completeness of follow-up make selection and information bias unlikely.

Our results were adjusted for many possible confounders, but residual confounding by unmeasured factors may still occur. No data on H. Pylori infection were collected at baseline in 1986 and, therefore, we could not adjust for this factor. In a Dutch study published in 2013, the seroprevalence of $H$. Pylori was estimated to be $48 \%$ among Dutch blood donors born between 1935 and 1946 and 16\% among those born between 1977 and 1987, indicating a birth-cohort effect [36]. Because the participants in our study were born between 1916 and 1932, we expect the prevalence in our study population to be higher than $48 \%$. H. Pylori infection has been shown to increase GNCA risk, whereas it decreases EAC risk and possibly also GCA risk in low-risk settings $[37,38]$. Therefore, H. Pylori infection may be a confounder if it is also associated with nut intake. If $H$. Pylori infection would be related to a reduced nut intake because of stomach complaints or decreased appetite, then the inverse association between nut intake and GNCA risk might be an overestimation. However, no evidence regarding this association is available. In addition, in recent years, several publications have hypothesized that statin use reduces esophageal and gastric cancer risk [39-41]. We recommend future studies to take these factors into account as well.

In conclusion, increased nut consumption was associated with a reduced risk of GNCA in this large prospective cohort study, and possibly with a reduced risk of ESCC. Peanut butter was not significantly related to gastric or esophageal cancer risk.

Acknowledgements We are indebted to the participants of this study and further wish to thank the cancer registries (IKA, IKL, IKMN, IKN, IKO, IKR, IKST, IKW, IKZ and VIKC), and the Netherlands nationwide registry of pathology (PALGA). We also thank Dr. A. Volovics and Dr. A. Kester for statistical advice; Dr. L. Schouten, S. van de Crommert, H. Brants, J. Nelissen, C. de Zwart, M. Moll, W. van Dijk, M. Jansen, and A. Pisters for assistance; and H. van Montfort, T. van Moergastel, L. van den Bosch, and R. Schmeitz for programming assistance.

Funding This study was funded by the Dutch Cancer Society (Grant number UM 2015-7860 to P.A. van den Brandt)

\section{Compliance with ethical standards}

Conflict of interest The authors declare that they have no conflict of interest.

Human rights statement All procedures followed were in accordance with the ethical standards of the responsible committee on human experimentation (institutional and national) and with the Helsinki Declaration of 1964 and later versions. Informed consent to be included in the study, or the equivalent, was obtained from all patients.

Open Access This article is distributed under the terms of the Creative Commons Attribution 4.0 International License (http://creativeco mmons.org/licenses/by/4.0/), which permits unrestricted use, distribution, and reproduction in any medium, provided you give appropriate credit to the original author(s) and the source, provide a link to the Creative Commons license, and indicate if changes were made.

\section{References}

1. van den Brandt PA, Schouten LJ. Relationship of tree nut, peanut and peanut butter intake with total and cause-specific mortality: a cohort study and meta-analysis. Int J Epidemiol. 2015;44(3):1038-49.

2. Aune D, Keum N, Giovannucci E, Fadnes LT, Boffetta P, Greenwood DC, et al. Nut consumption and risk of cardiovascular disease, total cancer, all-cause and cause-specific mortality: a systematic review and dose-response meta-analysis of prospective studies. BMC Med. 2016;14(1):207.

3. Wu L, Wang Z, Zhu J, Murad AL, Prokop LJ, Murad MH. Nut consumption and risk of cancer and type 2 diabetes: a systematic review and meta-analysis. Nutr Rev. 2015;73(7):409-25.

4. Chen GC, Zhang R, Martinez-Gonzalez MA, Zhang ZL, Bonaccio $M$, van Dam RM, et al. Nut consumption in relation to all-cause and cause-specific mortality: a meta-analysis 18 prospective studies. Food Funct. 2017. https://doi.org/10.1039/c7fo00915a.

5. Falasca M, Casari I, Maffucci T. Cancer chemoprevention with nuts. J Natl Cancer Inst. 2014;106(9):dju238.

6. Gonzalez CA, Salas-Salvado J. The potential of nuts in the prevention of cancer. Br J Nutr. 2006;96(Suppl 2):S87-94.

7. Ferlay J, Soerjomataram I, Dikshit R, Eser S, Mathers C, Rebelo $\mathrm{M}$, et al. Cancer incidence and mortality worldwide: sources, methods and major patterns in GLOBOCAN 2012. Int J Cancer. 2015;136(5):E359-86.

8. Domper Arnal MJ, Ferrandez Arenas A, Lanas Arbeloa A. Esophageal cancer: risk factors, screening and endoscopic treatment in Western and Eastern countries. World J Gastroenterol. 2015;21(26):7933-43.

9. Rustgi AK, El-Serag HB. Esophageal carcinoma. N Engl J Med. 2014;371(26):2499-509.

10. Devesa SS, Blot WJ, Fraumeni JF Jr. Changing patterns in the incidence of esophageal and gastric carcinoma in the United States. Cancer. 1998;83(10):2049-53.

11. Steevens J, Botterweck AA, Dirx MJ, van den Brandt PA, Schouten LJ. Trends in incidence of oesophageal and stomach cancer subtypes in Europe. Eur J Gastroenterol Hepatol. 2010;22(6):669-78.

12. Hashemian M, Murphy G, Etemadi A, Dawsey SM, Liao LM, Abnet CC. Nut and peanut butter consumption and the risk of esophageal and gastric cancer subtypes. Am J Clin Nutr. 2017. https://doi.org/10.3945/ajen.117.159467. 
13. Trichopoulos D, Ouranos G, Day NE, Tzonou A, Manousos O, Papadimitriou C, et al. Diet and cancer of the stomach: a casecontrol study in Greece. Int J Cancer. 1985;36(3):291-7.

14. Wang XQ, Yan H, Terry PD, Wang JS, Cheng L, Wu WA, et al. Interaction between dietary factors and Helicobacter pylori infection in noncardia gastric cancer: a population-based case-control study in China. J Am Coll Nutr. 2012;31(5):375-84.

15. Hoshiyama Y, Sasaba T. A case-control study of stomach cancer and its relation to diet, cigarettes, and alcohol consumption in Saitama Prefecture, Japan. Cancer Causes Control. 1992;3(5):441-8.

16. Hoshiyama Y, Sasaba T. A case-control study of single and multiple stomach cancers in Saitama Prefecture, Japan. Jpn J Cancer Res. 1992;83(9):937-43.

17. Zhao Y, Zhao L, Hu Z, Wu J, Li J, Qu C, et al. Peanut consumption associated with a reduced risk of esophageal squamous cell carcinoma: a case-control study in a high-risk area in China. Thorac Cancer. 2017. https://doi.org/10.1111/1759-7714.12520.

18. Samoli E, Lagiou A, Nikolopoulos E, Lagogiannis G, Barbouni A, Lefantzis D, et al. Mediterranean diet and upper aerodigestive tract cancer: the Greek segment of the alcohol-related cancers and genetic susceptibility in Europe study. Br J Nutr. 2010;104(9):1369-74.

19. van den Brandt PA, Goldbohm RA, van't Veer P, Volovics A, Hermus RJ, Sturmans F. A large-scale prospective cohort study on diet and cancer in The Netherlands. J Clin Epidemiol. 1990;43(3):285-95.

20. Van den Brandt PA, Schouten LJ, Goldbohm RA, Dorant E, Hunen PM. Development of a record linkage protocol for use in the Dutch Cancer Registry for Epidemiological Research. Int J Epidemiol. 1990;19(3):553-8.

21. Goldbohm RA, van den Brandt PA, Dorant E. Estimation of the coverage of Dutch municipalities by cancer registries and PALGA based on hospital discharge data. Tijdschr Soc Gezondheidsz. 1994;72:80-4.

22. Goldbohm RA, van den Brandt PA, Brants HA, van't Veer P, Sturmans F, et al. Validation of a dietary questionnaire used in a large-scale prospective cohort study on diet and cancer. Eur J Clin Nutr. 1994;48(4):253-65.

23. Lin DY, Wei L-J. The robust inference for the Cox proportional hazards model. J Am Stat Assoc. 1989;84(408):1074-8.

24. Schoenfeld D. Partial residuals for the proportional hazards regression model. Biometrika. 1982;69(1):239-41.

25. de Vogel S, Bongaerts BW, Wouters KA, Kester AD, Schouten LJ, de Goeij AF, et al. Associations of dietary methyl donor intake with MLH1 promoter hypermethylation and related molecular phenotypes in sporadic colorectal cancer. Carcinogenesis. 2008;29(9):1765-73.

26. Wacholder S, Gail MH, Pee D, Brookmeyer R. Alternative variance and efficiency calculations for the case-cohort design. Biometrika. 1989;76(1):117-23.
27. Akaike $\mathrm{H}$. A new look at the statistical model identification. IEEE Trans Automat Control. 1974;AC-19:716-23.

28. Fung TT, McCullough ML, Newby PK, Manson JE, Meigs JB, Rifai N, et al. Diet-quality scores and plasma concentrations of markers of inflammation and endothelial dysfunction. Am J Clin Nutr. 2005;82(1):163-73.

29. Botterweck AA, van den Brandt PA, Goldbohm RA. A prospective cohort study on vegetable and fruit consumption and stomach cancer risk in The Netherlands. Am J Epidemiol. 1998;148(9):842-53.

30. Willett WC. Nutritional epidemiology. 2nd ed. New York: Oxford University Press; 1998.

31. Ros E. Health benefits of nut consumption. Nutrients. 2010;2(7):652-82.

32. Stichting Nederlands Voedingsstoffenbestand. NEVO-table. Dutch Food Composition Table 1986-1987, Nederlands voedingsstoffenbestand. The Hague, The Netherlands: Voorlichtingsbureau voor de Voeding; 1986.

33. McColl KE, Going JJ. Aetiology and classification of adenocarcinoma of the gastro-oesophageal junction/cardia. Gut. 2010;59(3):282-4.

34. Schouten LJ, Jager JJ, van den Brandt PA. Quality of cancer registry data: a comparison of data provided by clinicians with those of registration personnel. Br J Cancer. 1993;68(5):974-7.

35. Figures on cancer (Cijfers over Kanker) [Internet]. 2016 [cited 27-10-2017]. Available from: http://www.cijfersoverkanker.nl/ meest-voorkomende-soorten-52.html.

36. van Blankenstein $\mathrm{M}$, van Vuuren AJ, Looman CW, Ouwendijk M, Kuipers EJ. The prevalence of Helicobacter pylori infection in the Netherlands. Scand J Gastroenterol. 2013;48(7):794-800.

37. Xie FJ, Zhang YP, Zheng QQ, Jin HC, Wang FL, Chen M, et al. Helicobacter pylori infection and esophageal cancer risk: an updated meta-analysis. World J Gastroenterol. 2013;19(36):6098-107.

38. Cavaleiro-Pinto M, Peleteiro B, Lunet N, Barros H. Helicobacter pylori infection and gastric cardia cancer: systematic review and meta-analysis. Cancer Causes Control. 2011;22(3):375-87.

39. Thomas T, Loke Y, Beales ILP. Systematic review and metaanalysis: use of statins is associated with a reduced incidence of oesophageal adenocarcinoma. J Gastrointest Cancer. 2017. https ://doi.org/10.1007/s12029-017-9983-0.

40. Singh PP, Singh S. Statins are associated with reduced risk of gastric cancer: a systematic review and meta-analysis. Ann Oncol. 2013;24(7):1721-30.

41. Wu XD, Zeng K, Xue FQ, Chen JH, Chen YQ. Statins are associated with reduced risk of gastric cancer: a meta-analysis. Eur J Clin Pharmacol. 2013;69(10):1855-60. 